\title{
MICROSCOPÍA ELECTRÓNICA DE BARRIDO DE LA HIPERPLASIA EN LA VELLOSIDAD MOLAR
}

\author{
Olivar Castejón S. ${ }^{1}$, Oliver Castejón M. ${ }^{a}$ \\ 1 Laboratorio de Microscopía Electrónica, Facultad de Ciencias de la Salud, Universidad de Carabobo - Núcleo Aragua, \\ Centro de Investigación y Análisis Docente Asistencial del Núcleo Aragua (CIADANA), Maracay, Aragua, Venezuela.
}

${ }^{\text {a }}$ Alumno, Escuela de Medicina, Facultad de Ciencias de la Salud, Universidad de Carabobo - Núcleo Aragua, Venezuela.

\section{RESUMEN}

Objetivo: Estudio de la superficie externa del sincitiotrofoblasto de vesículas de mola hidatidiforme, fue rastreada utilizando la microscopía electrónica de barrido. Método: Especimenes de material molar de 21 semanas de embarazo se fijaron en $2 \%$ de glutaraldehido $0,1 \mathrm{M}$ a $4^{\circ} \mathrm{C}$ en sala de parto y posteriormente post fijados en $1 \%$ de tetraoxido de osmio siguiendo los procedimientos convencionales de la microscopía electrónica de barrido como deshidratación, desecado de punto crítico, cubrimiento iónico y observación con el microscopio electrónico de barrido. Resultados: Los resultados revelan cambios morfológicos en la membrana plasmática sincitial, desde superficie aplanadas con pequeños gránulos o promontorios, hasta la formación de numerosas prolongaciones de membrana que originan pliegues, bandas o columnas, las cuales se ramifican intensamente, tomando contacto entre sí para conformar una complicada trama, que deja un retículo de espacios como cavernas, las cuales se abren hacia el espacio intervelloso siendo la expresión de una intensa proliferación de membranas en hiperplasia. Conclusión: La observación de esta trama permite un mejor entendimiento de la estructura de la mola comparada con las imágenes de microscopía de luz.

\section{PALABRAS CLAVE: Microscopía electrónica de barrido, hiperplasia placentaria, trofoblasto, mola hidatidiforme}

\section{SUMMARY}

Objective: Study of the external surface of the syncytiotrophoblast in vesicles of hydatidiform mole was examined and analysed using scanning electron microscopy. Method: Vesicles of molar material were taken at 21 weeks of pregnancy and fixed in $2 \%$ of glutaraldehyde $0.1 \mathrm{M}$ at $4{ }^{\circ} \mathrm{C}$ in delivery room and furtherly post-fixed in $1 \%$ of tetroxide of osmium according with conventional procedures of the scanning electron microscopy as dehydration, critical point drying, surface coating and examination using scanning electron microscope. Result: The findings reveal the morphological changes in the syncytial membrane from smooth surface with small bridges or granules to the formation of numerous prolongations of plasma membrane, which produce folds, bands or columns with ramifications, that get in touch organizing a complex net that contain spaces opened to the intervillous space. This is an expression of the proliferation of syncytial plasma membrane during the hyperplasia. Conclusion: The tridimensional observation of this net complex permit a better understanding of the structure of the molar vesicle when these images are compared with those of light microscopy.

KEY WORDS: Scanning electron microscopy, placental hyperplasia, trophoblast, hydatidiform mole 


\section{INTRODUCCIÓN}

La microscopía electrónica de barrido (MEB) ha sido muy poca aplicada al estudio de la mola hidatidiforme, la cual ha sido tratada principalmente en su aspecto anatomopatológico (1), epidemiológico (2), genético (3), histopatológico y clínico (4), siendo la hiperplasia trofoblástica lo que diferencia la mola hidatidiforme de otros procesos placentarios acompañados de cambios edematosos. La aplicación de esta técnica microscópica a la anatomía patológica de la vellosidad molar, pudiera brindar un mejor entendimiento de cómo los procesos de invasión, migración y proliferación se realizan siguiendo una visión de conjunto más completa, con mayor profundidad de campo o más real, que la simple línea festoneada que bordea a la membrana plasmática del trofoblasto en un corte bidimensional de 4 a 5 um, lograda con los cortes y tinciones de hematoxilina y eosina (H\&E) de la microscopía de luz (ML).

Hay problemas y fallas en el diagnóstico histopatológico (5). Los diversos cambios morfológicos que afectan la membrana plasmática del sincitiotrofoblasto en los procesos de hiperplasia no pueden ser observados en su totalidad. Una visión parcializada de la hiperplasia con la ML no permite al patólogo el mejor conocimiento del pleomorfismo celular existente. La hiperplasia ha sido un elemento esencial para establecer el diagnóstico de mola hidatidiforme utilizado para describir la enfermedad trofoblástica gestacional.

Acumulación de vacuolas citoplasmáticas, intensa deposición de glucógeno y amplias regiones dilatadas de retículo endoplásmico rugoso son comunes en hiperplasia de humanos y ratones (6). Se requieren nuevos criterios adaptables a la diferenciación de mola completa y aborto hidrópico ya que existe variabilidad inter e intra observador en el diagnóstico de mola hidatidiforme (7). Crecimiento trofoblástico polar visto en aborto hidrópico pudiera también verse en mola parcial. La MEB contribuiría en nuestro estudio con esta problemática. La importancia de la investigación radica en que hay condiciones extremadamente raras donde la hiperplasia trofoblástica se vuelve polimórfica y persistente, llegando a sufrir transformación maligna que invade al miometrio (8). El objetivo de este estudio es describir con la MEB los cambios morfológicos de la membrana plasmática del sincitio en las regiones correspondiente a la zona de hiperplasia del trofoblasto.

\section{MATERIAL Y MÉTODO}

Especímenes de 50 vesículas fueron obtenidos para el análisis de la superficie externa en cada una de ellas, de paciente con embarazo molar completo de 21 semanas, sin estudio genético, fijados en $2 \%$ de glutaraldehido $0,1 \mathrm{M}$ a $4^{\circ} \mathrm{C}$, en sala de parto, posteriormente lavados y post fijados en $1 \%$ de tetraóxido de osmio por 2 horas a la misma temperatura. Material de placenta normal fue obtenido como control de la misma manera. Ambos materiales siguieron los procedimientos convencionales de la microscopía electrónica de barrido (9), tales como deshidratación, desecado de punto crítico en desecador HCP2, Hitachi (Tokyo CO, LTD, Japón) cubiertos con platino $(\mathrm{Pt})$ y paladio $(\mathrm{Pd})$ en cobertor iónico, Eiko IB3 (CO, LTD, Tokyo, Japón) y examinados en un MEB Hitachi S2300 (Nissei Sangyo, CO, LTD, Tokio, Japón).

\section{RESULTADOS}

Al rastrear la superficie externa de las vesículas molares de regiones correspondientes a la hiperplasia se notan en algunos lugares, sinuosas elevaciones similares a un sistema de montañas con ligeros promontorios sobre la superficie. Esta se mostró arrugada, la cual presenta en algunas zonas microvellosidades degeneradas y pequeñas depresiones o huecos en la superficie (Figura 1). En otras regiones los montículos se hacen más prominentes y se ramifican. La superficie se mostró conformada por cilindros semicortados que se extienden y desplazan dando el aspecto de pliegues continuos (Figura 2). Grandes prolongaciones de membrana se elevan sobre la superficie dejando espacios entre ellas. Numerosas extensiones de membranas alargadas, piriformes $u$ ovoides sobresalen hacia el espacio intervelloso (Figura 3).

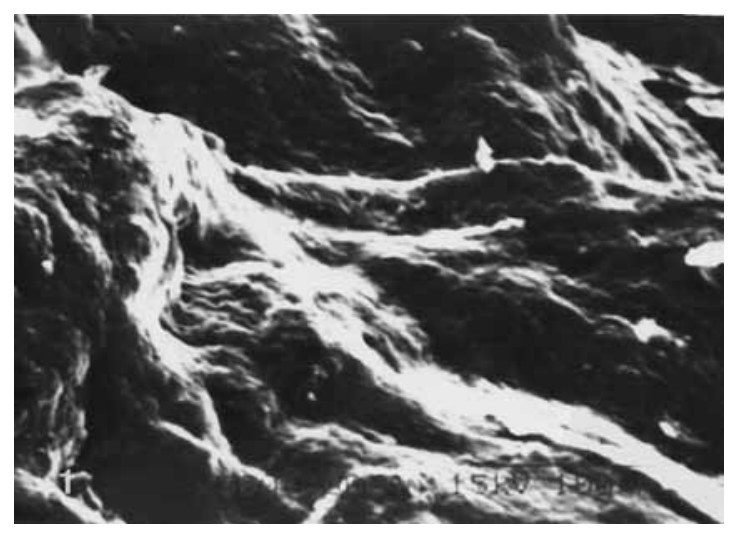

Figura 1. Región de superficie de vesícula molar, mostrando suaves elevaciones de membrana con pequeños abultamientos. 


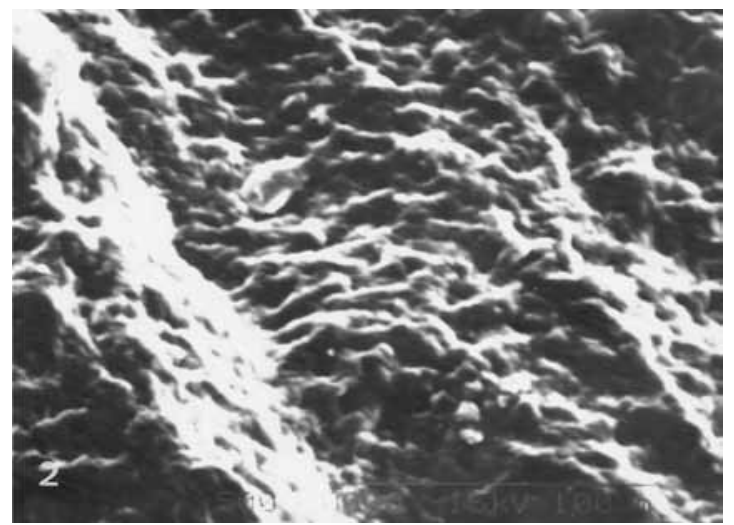

Figura 2. La superficie muestra múltiples pliegues ramificados de membrana que sobresalen hacia el espacio intervelloso.

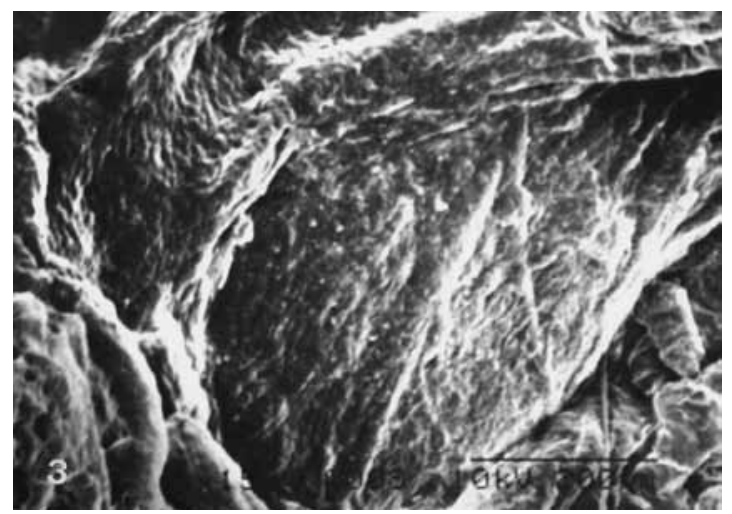

Figura 3. Los pliegues de membrana se han levantado a gran distancia de la superficie plana que se observa al centro con microvellosidades degeneradas.

Las prolongaciones en forma de túbulos semicortados se entrecruzan (Figura 4) y toman contacto entre ellas. La elevación de las membranas sobre la superficie va dejando espacios que se comunican y conforman una complicada red de túneles. Brotes sincitiales de aspecto piriforme se observan indicando la proliferación de vellosidades nuevas. La superficie se nota en algunas regiones cubiertas por fibrina. Zonas irregulares de membrana han dejado amplios espacios similares a cavernas (Figura 5). La hiperplasia se hace tan compleja que toma el aspecto de extensos pliegues semicirculares que sobresalen de la superficie para tomar contactos unos con otros (Figura 6). En aquellos espacios de la trama pueden encontrarse gran cantidad de eritrocitos, estando "in vivo", cuando son observados con ML. La trama conformada por varias prolongaciones ramificadas de membranas sincitiales que toman contacto sobre la superficie han originado, finalmente, una red de espacios o canales con comunicación hacia el exterior. La superficie de las membranas sincitiales ha venido invadiendo la zona correspondiente al espacio intervelloso con un exagerado crecimiento, hasta formar esa estructura tridimensional de canales o espacios abiertos (Figura 7).

\section{DISCUSIÓN}

Una visión tridimensional de la superficie externa al trofoblasto que muestra una trama de columnas, bandas y plegamientos, que conforman una red que se comunica con espacios abiertos hacia el espacio intervelloso, ha sido mostrada.

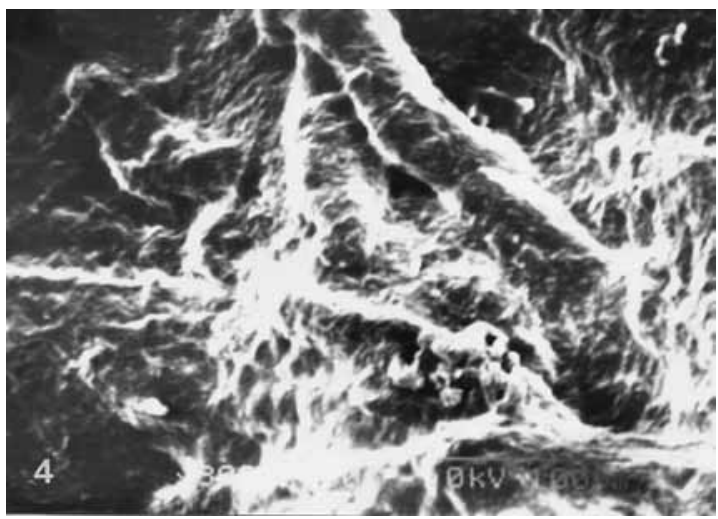

Figura 4. Los pliegues se entrecruzan y toman contacto unos sobre otros. Depresiones o excavaciones de la superficie se observan en la microfotografía.

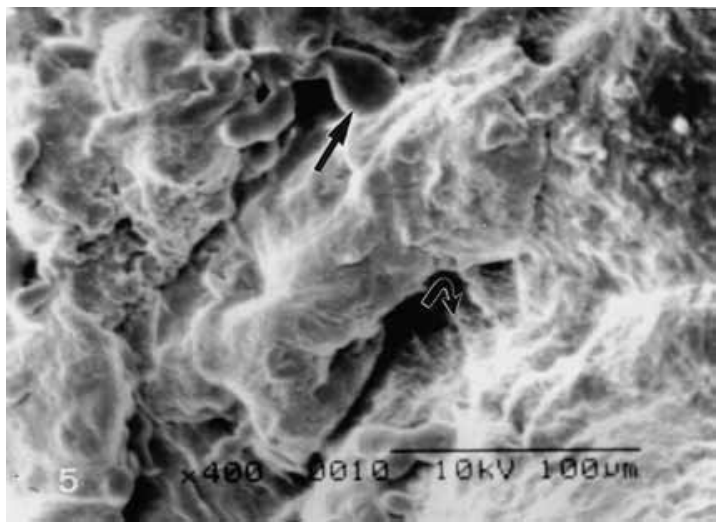

Figura 5. La flecha señala un brote del sincitio. Una banda plegada irregular sobrepasa a una superficie trofoblástica dejando un espacio abierto (flecha curva). 


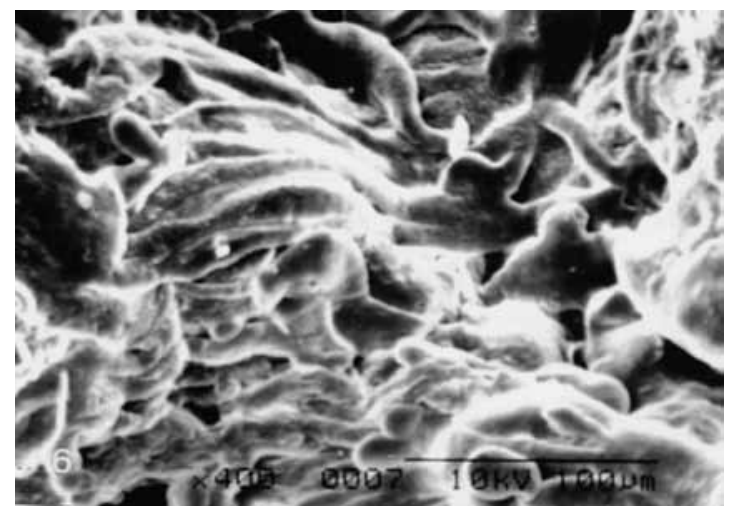

Figura 6. Numerosos plegamientos de membranas muestran la exacerbada proliferación de membranas o hiperplasia en la región superficial de la vesícula molar.

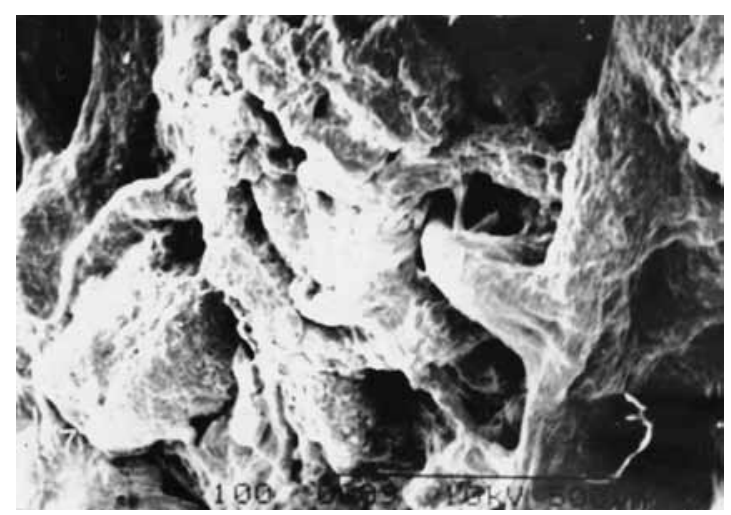

Figura 7. Columnas de membranas sobresalen en la superficie para asociarse unas con otras y formar una red tridimensional que deja espacios abiertos.

Ockleford y cols $(9,10)$, utilizando MEB describen de la misma manera, los pliegues de membrana observados en este trabajo. Como también los brotes sincitiales, los orificios o las aberturas de más de $750 \mathrm{~nm}$ que comunican con canales, y un patrón reticular de bandas de gránulos de 2-4 um que conforman estructuras poligonales en la superficie del trofoblasto, cuyos espacios encerrados varían de tamaño; microvellosidades alargadas que terminan en puntas bulbosas y microgibosidades que son grupos de microvillosidades extremadamente alargadas de la superficie de la membrana plasmática. Estas observaciones coinciden con nuestros resultados en los pequeños abultamientos y brotes sincitiales.

Suponemos que una gran diversidad de estructuras organizadas en la superficie del trofoblasto suelen desarrollarse en cada una de las vesículas observadas, según sea el sobrecrecimiento de las proteínas estructurales poliméricas corticales, asociadas a la membrana plasmática, que le dan soporte a ésta (9). En previos estudios (11), pliegues de trofoblasto se corresponden con bandas o montículos de la microscopía de luz. Las proteínas estructurales de la capa cortical del sincitio han perdido el control genético de su producción debido al desequilibrio genético que ocurre en estas anomalías de origen cromosómico. Anteriormente se interpretaba que los numerosos eritrocitos incluidos en el interior de la trama formaban parte del material alimenticio en la incrementada actividad del trofoblasto. Esta nueva visión permite sugerir una entrada y salida de sangre materna por los espacios o canales que quedan abiertos hacia el espacio intervelloso.

La hiperplasia se relaciona con la membrana basal, que sostiene al sincitio o citotrofoblasto, organización especializada como hoja de proteínas y de glucosaminoglucanos de la matriz extracelular (12). Cambios degenerativos que ocurren en ella, permiten que el trofoblasto se separe e invada el espacio intervelloso (13). La pérdida de colágeno IV, laminina P-1 y laminina M desestabilizan la membrana basal del trofoblasto molar y este puede adquirir propiedades invasoras en la transformación maligna (14).

La resistencia a la acción del factor transformador del crecimiento tipo B (T6FB), que impide la proliferación, migración e invasión de estas células sería la responsable de la transformación maligna por una activación de los oncogenes (15). En la maquinaria del ciclo celular, implicada en la transformación maligna del trofoblasto, la proteína ciclina $E$, que se une y activa a varias quinasas, formando complejos activadores de los genes iniciadores de la replicación del DNA, ha sido encontrada con una mayor expresión en coriocarcinoma y menor en mola hidatidiforme con las técnicas de inmunohistoquímica (16).

Moléculas que han tenido una marcada importancia en los procesos de diferenciación celular como el receptor activador-proliferador del peroxisoma (PPAR), receptor hormonal nuclear, está disminuido en mola y casi negativo en el coriocarcinoma, indicando rasgos en las células de indiferenciación celular, lo que es típico de procesos neoplásicos $(17,18)$. Proteoglucanos conocidos como sindecanos asociados a proteasas o antiproteasas, tienen un papel que pudiera explicar la conducta invasiva de estos trofoblasto, observándose que la carencia de esa asociación, en parte pudiera explicar, la invasividad (19). 
Esta hiperplasia que es capaz de provocar invasión hacia otros tejidos, no ha podido ser considerada como predictiva hacia enfermedad trofoblástica persistente en material molar. Cuando mediante técnicas de inmunohistoquímica, utilizando el marcador de proliferación celular Ki67, después de la evacuación en casos de mola completa, seguida de enfermedad trofoblástica gestacional persistente y casos que se resolvieron espontáneamente, se pudo detectar que no hubo diferencias significativas en los índices de Ki67 tanto del trofoblasto extravelloso como el citotrofoblasto velloso. No hay parámetros clínicos ni histopatológicos confiables para predecir el desarrollo de enfermedad trofoblástica gestacional persistente después de la evacuación (20), siendo los valores séricos de gonadotropina coriónica el único factor confiable.

No hemos pretendido, con imágenes de MEB estáticas, dar una explicación de los factores etiológicos que promueven la hiperplasia. Como se comprenderá, ésta ha sido interpretada en base a los avances de la biología molecular. Se ha mostrado como la superficie del trofoblasto se expande intensamente formando una estructura de columnas forradas de trofoblasto que se interconectan entre sí, para formar un enrejado por donde circula sangre materna "in vivo".

Esta hiperplasia vista acá, también puede ser observada en abortos espontáneos, en vellosidades no hidrópicas, en trisomías 7, 15, 21 y 22 (21). Moderada a abundante hiperplasia trofoblástica y lagunas trofoblásticas asociadas a vesículas molares con lagunas estromales hidrópicas son características predictivas de una identificación de triploidia (22).

\section{CONCLUSIÓN}

Una nueva imagen tridimensional mostrada con microscopía electrónica de barrido de la hiperplasia del trofoblasto, en la superficie de la vesícula molar ha sido presentada, la cual permite un mejor entendimiento de la organización de la membrana celular cuando se compara con la suministrada por la microscopía de luz.

\section{BIBLIOGRAFÍA}

1. De Castillo JP, De Uzcategui MLC, Moreno F, Tovitto G. Estudio anatomopatológico de un caso de mola parcial. Rev Obstet Ginecol Venez 2006;66:107-17.

2. Dreyfus M, Tissier I, Philippe E. Gestational trophoblastic diseases classification, epidemiology and genetic data. J Gynecol Obstet Biol Reprod 2000;29:6879.

3. Kato HD, Terao Y, Ogawa M, Matsuda T, Arima T,
Kato $\mathrm{K}$, et al. Growth-associated gene expression profiles by microarray analysis trophoblast of molar pregnancies and normal villi. Int J Gynecol Pathol 2002;21:255-60.

4. Moher R, Goldstein DP, Berkowitz R, Berstein M, Genetest DR. Complete hydatidiform mole. Comparison of clinicopathologic features, current and past. J Reprod Med 1998;43:21-7.

5. Discoll SG. Problems and pitfalls in the histopathologic diagnosis of gestational trophoblastic lesions. J Reprod Med 1987;32:623-8.

6. Wakisaka N, Inoue K, Ogunuki N, Miki H, Sekita Y, Hanaki K, et al. Ultrastructure of placental hyperplasia in mice: comparison of placental phenotypes with three different etiologies. Placenta 2008;29:753-9.

7. Fukunaga $M$, Katabuchi $H$, Nagasaka $T$, Mikami $Y$, Minamiguchi S, Lage JM. Interobserver and intraobserver variability in the diagnosis of hydatidiform mole. Am J Surg Pathol 2005;29:942-7.

8. Makovitzky J, Vogel M, Miessner R, Mylonas I, VogtWeber B, Richter DU. Diagnostic aspects of hydatidiform mole with persistence of polymorphic trophoblastic hyperplasia. Anticancer Res 2003;23:1069-73.

9. Ockleford C, Barker C, Griffiths J, Mcturk G, Fisher R, Lawler S. Hydatidiform Mole: an ultrastructural analysis of syncytiotrophoblast surface organization. Placenta 1989;10:195-212.

10. Ockleford CD, Clode A. Microgibbosities in hydatidiform mole. J Pathol 1983;2:181-9.

11. Castejón OC, Molina VR, Rivas A AE, Aguirre O, Graterols IJ. La variabilidad morfológica de la vellosidad placentaria hidrópica. Gac Med Caracas 2002;110:210-6.

12. Castejón OC. La lámina basal trofoblástica y su relación con la ultraestructura del trofoblasto. Gac Med Caracas 2005;113:65-71.

13. Scucces MG, Castejón OC. Lesiones predictivas de malignidad en la membrana basal de mola hidatidiforme. Rev Obstet Ginecol Venez 2006;66:151-8.

14. Castejón OC, Scucces MG, Rivas AE, Graterol IJ. La membrana basal en caso de mola hidatidiforme completa. Rev Obstet Ginecol Venez 2002;62:207-13.

15. Lala PK, Khoo KS, Guimond MJ, Chakraborty Ch. Control mechanisms in human trophoblast proliferation and invasiveness: then derangement during trophoblastic tumor progression. Trophoblast Research 1999;13:119-36.

16. Kim YT, Cho NH, Ko JH, Yang WI, Kim JW, Choi EK, et al. Expression of cyclin $\mathrm{E}$ in placentas with hydropic change and gestational trophoblastic diseases. Implications for the malignant transformation of trophoblasts. Cancer 2000;89:673-9.

17. Capparuccia L, Marzioni D, Giordano A, Fazicli F, De Victolis $\mathrm{M}$, Busso $\mathrm{M}$, et al. PPARy expression in normal human placenta, hydatidiform mole and choriocarcinoma. Mol Hum Reprod 2002;8:574-9.

18. Scucces M. Algunas consideraciones acerca de la biología del trofoblasto en la mola hidatidiforme. Rev Obstet Ginecol Venez 2008;68:98-104.

19. Crescimanno C, Marzioni D, Paradinas FJ. Schrurs $\mathrm{B}$, Muhlhauser J, Todros $\mathrm{T}$, et al. Expression pattern alterations of syndecans and glypican-1 in normal and 
pathological trophoblast. J Pathol 1999;189:600-8.

20. Jeffers MD, Richmond JA, Smith R. Trophoblast proliferation rate does not predict progressions to persistent gestational trophoblastic disease in complete hydatidiform mole. Int J Gynecol Pathol 1996;15:34-8.

21. Redline RW, Hassold T, Zaragoza M. Determinants of villous trophoblastic hyperplasia in spontaneous abortions. Mod Pathol 1998;8:762-8.
22. Van Lijnschoten G, Arends JW, Leffers $P$, De La Fuente HA, Van Der Looij HJ, Geraedts JP. The value of histomorphological features of chorionic villi in early spontaneous abortion for the prediction of karyotype. Histopathology 1993;22:557-63. 